\title{
El modelo educativo en México: una revisión de su alcance y una perspectiva para el futuro
}

\author{
Aleida Azamar-Alonso*
}

Maestría en Estudios Latinoamericanos. Profesora, Universidad Autónoma Metropolitana, Unidad Xochimilco, Distrito Federal, México.

Correo electrónico:

gioconda15@gmail.com

Recibido: 21 de mayo del 2015

Aprobado: 10 de agosto del 2015

Cómo citar este artículo: Azamar-Alonso, Aleida. "El modelo educativo en México: una revisión de su alcance y una perspectiva para el futuro". Rastros Rostros 17.31 (2015): 127-141. Impreso. doi: http://dx.doi. org/10.16925/ra.v17i31.1094

\section{Resumen}

Propósito: el modelo educativo en México responde a intereses que no concuerdan con la realidad social, ya que no se garantiza la superación de la pobreza y la marginación con este modelo. En este artículo se estudia la formación histórica del proceso educativo en México, a partir de la década de los ochenta hasta el 2010. Descripción: de carácter explicativa, esta investigación se propone conocer la evolución de la estructura educativa de México, así como comprobar si su desarrollo concuerda con el objetivo económico presente en el modelo de desarrollo nacional. Por esta razón, la recolección de datos es de orden cualitativo y cuantitativo. Los datos fueron obtenidos del Sistema Nacional de Información Estadística Educativa y a través de publicaciones oficiales. Punto de vista: se exponen las propuestas del movimiento de desescolarización, con la finalidad de elaborar una perspectiva crítica que permita determinar si el modelo educativo aplicado actualmente responde a los intereses sociales mayoritarios o si, por el contrario, requiere una reforma basada en las ideas propuestas por autores como Ilich, McLuhan o Freire, según las cuales los individuos y la sociedad se encargan del proceso educativo a través de redes y organismos no centralizados u orientados hacia fines técnicos y económicos. Conclusiones: se puede observar un modelo educativo incapaz de responder a la demanda, desfasado en el tiempo y nulo en utilidad ante los nuevos paradigmas sociales, por lo que es necesario hacer un análisis de fondo sobre los objetivos que se pretenden cumplir.

Palabras clave: desescolarización, educación, formación técnica, pobreza. 


\title{
The Educational Model in Mexico: A Review of its Scope and a Perspective for the Future
}

\begin{abstract}
Purpose: the educational model in Mexico responds to an agenda that do not match social reality, since overcoming poverty and marginalization is not guaranteed with this model. This article analyzes the historical formation of the educational process in Mexico, from the eighties until 2010. Description: this research of explanatory nature aims to understand the evolution of the educational structure in Mexico and verify if its development is consistent with the economic objective included in the national development model. For this reason, data collection is both qualitative and quantitative. Data were obtained from the National Statistical Education Information System and through official publications. Point of view: the proposals of the unschooling movement are discussed in order to develop a critical perspective to determine whether the educational model now applied responds to the major social interests or whether, by contrast, it requires a reform based on the ideas proposed by authors like Ilich, McLuhan or Freire, according to which individuals and society are responsible for the educational process through non-centralized or technically/economically oriented bodies or networks. Conclusions: an educational model unable to respond to demand, behind the times, and with zero profit regarding new social paradigms is observed; therefore, it is necessary to analyze in depth the objectives to be met.
\end{abstract}

Keywords: unschooling, education, technical training, poverty.

\section{O modelo educativo no México: uma revisão do seu alcance e uma perspectiva para o futuro \\ Resumo}

Propósito: o modelo educacional no México responde a interesses que não coincidem com a realidade social, porque não se assegura a superação da pobreza e a marginação com esse modelo. Neste artigo estuda-se a formação histórica do processo educativo no México, a partir da década de oitenta e até o ano 2010. Descrição: de caráter explicativo, esta investigação propõe-se conhecer a evolução da estrutura educativa do México, bem como comprovar se seu desenvolvimento coincide com o escopo económico presente no modelo de desenvolvimento nacional. Por isso, a coleta de dados é de tipo qualitativo e quantitavo. Os dados foram obtidos do Sistema Nacional de Informação Estatística Educativa e através de publicações oficiais. Ponto de vista: propostas do movimento de desescolarização são expostas visando criar uma perspectiva crítica que permita determinar se o modelo educativo aplicado atualmente responde aos interesses sociais maioritários ou se pelo contrário, requer uma reforma baseada nas ideias propostas por autores como Ilich, McLuhan ou Freire, que propõem que os indivíduos e a sociedade se encarregam do processo educativo através de redes e organismos não centralizados ou voltados a fins técnicos e económicos. Conclusões: observase um modelo educativo incapaz de responder à demanda, desfasado no tempo e não é útil perante os novos paradigmas sociais; pelo que é necessário fazer uma análise de fundo sobre os objetivos pretendidos.

Palavras-chave: desescolarização, educação, formação técnica, pobreza. 
El modelo educativo en México se construye a partir del enfoque social e histórico del sistema de gobierno que se pretende implementar. Su uso es una esquematización de procesos basados en programas, los cuales recogen y simplifican la vasta cantidad de estudios pedagógicos adecuados para la orientación en la que se intenta educar (De Zubiría).

La labor educativa pretende ser una adecuación del individuo con miras a su integración al sistema económico. A los alumnos se les educa como parte de un todo, bajo la suposición según la cual están obligados a circunscribirse a las necesidades de un sistema político que les beneficia con el acceso a una institución (Ratinoff). Sin embargo, en muchas ocasiones dicha preparación cuenta con diversos problemas heredados desde su fundación, y en su ejecución termina por generar varios enfrentamientos con el medio social en el que se instala.

Los organismos gubernamentales encargados de sintetizar el modelo educativo para su masificación a través de programas de estudio, no consideran la amplia diversidad de identidades sociales y económicas en las cuales se implementan los programas. Suponen escenarios en los que no se enfrentan dichas disparidades, por lo que requerimientos tales como códigos de vestimenta generalizados, uso de materiales didácticos obligatorios, etc., los cuales supuestamente sirven para conducir de manera "correcta" el proceso de formación educativa, suelen ser inoperantes en zonas donde la marginación social y el empobrecimiento afectan el nivel de vida de los estudiantes.

A fin de operar a nivel efectivo un programa educativo, este debe impartirse gradualmente desde la niñez —en forma de educación básica - hasta la edad adulta, y de forma más completa y profunda, la formación profesional. Este proceso de modelamiento ininterrumpido está planeado en México para concluirse en un lapso de aproximadamente 18 años. Por esto, resultaría complicado elaborar una reforma educativa radical que se adapte a los distintos niveles al proponer nuevos objetivos básicos, ya que existe una amplia base de estudiantes que se encuentran en activo y estos cambios afectarían gravemente la continuidad del proceso y el objetivo propuesto.

En este procedimiento iterativo académico no se contemplan espacios de tiempo diferenciados en las distintas ramas de la formación educativa. Es decir, no hay una explicación concreta de la razón por la que se requiere la misma cantidad de tiempo para la formación de estudiantes cuyo interés sea la rama humanista, como para la de estudiantes que busquen formarse como ingenieros especialistas en la construcción de motores de maquinaria.

Los procesos temporales de avance técnico en el mercado laboral superan ampliamente los niveles de tiempo del avance académico. Es decir, la escuela como entidad encargada de la formación y la vanguardia está desfasada a causa de los progresos técnicos de la vida profesional. Debido a que el plan educativo se plantea con base en las necesidades de un espacio de tiempo concreto, no se consideran las estimaciones de cambio en la población candidata a formarse, lo cual a menudo genera una demanda que no es posible cubrir: los planes de estudio están diseñados para el tratamiento de un grupo de estudiantes que no supere un número específico.

La escuela - como centro del conocimiento y de formación - se ve superada a nivel físico por la creciente demanda. No es una solución mantener a largo plazo una institución que puede privilegiar la formación solo de unos cuantos. La inversión económica requerida para la ampliación de la matrícula estudiantil es considerablemente alta y requiere de tiempo, de manera que esto afecta negativamente a los estudiantes rechazados en los procesos de elección para ingresar a los niveles más altos de formación académica.

En razón a la alta tasa de deserción de los estudiantes, la inversión económica en la educación se critica por lo ineficaz que resulta en su planteamiento. Estos problemas pueden explicarse más por una cuestión del modelo educativo/pedagógico aplicado que por las mismas características de los estudiantes. Si se parte de este escenario, las políticas económicas afectan profundamente el desarrollo del proceso de creación del modelo educativo; en este el conocimiento se vincula principalmente al servicio del aparato productivo, y como adecuación a esa situación todo conocimiento emanado debe ser práctico, debe dejar de lado los principios de crítica y juicio humano (Luengo et al.).

$\mathrm{Si}$, por lo tanto, la productividad educativa actualmente se mide en términos de beneficio económico, la hipótesis es entonces la siguiente: a través de la modernización del proceso de formación educativa se crean las condiciones para mejorar el desempeño productivo de un país (OCDE).

Dicha modernización académica se orienta en disposición de los acuerdos realizados con el Banco Mundial (Bм) y el Fondo Monetario Internacional (FMI), a fin de establecer guías que fomentan competencias laborales e integren procedimientos 
selectivos para el acceso a la formación educativa (Ratinoff).

Con base en lo comentado anteriormente, la estructura del trabajo se dividirá de la siguiente forma. En la primera parte, se expone la metodología utilizada para la recolección de datos estadísticos y su relevancia para el análisis. En la segunda parte, se realiza un breve repaso de las propuestas teóricas realizadas por los principales autores del movimiento de la desescolarización, revisando las proposiciones que hace cada uno de ellos sobre el problema del sistema educativo y cómo podría orientarse de forma más adecuada. La tercera sección se centra en la formación del proceso educativo en México, durante un periodo de 30 años, comenzando en 1980 y culminando en el 2010. En este apartado se presentan datos estadísticos que cubren la matrícula escolar para el nivel básico, medio superior y superior, además de la cantidad de profesores y la evolución en los niveles de escolarización en México. Por otra parte, se abordan los principios rectores con los que se inicia el proceso educativo nacional, así como los más influyentes actores que tuvieron relación con la formalización del actual modelo educativo. La cuarta sección explica la relación presente entre el proceso educativo y el problema de la pobreza entre la población. En el quinto apartado se revisan las propuestas emanadas desde los modelos de la desescolarización aplicadas al caso de México, una revisión de su posible aplicación y un análisis de las implicaciones que tendrían. Finalmente, se presentan las conclusiones del trabajo.

\section{Metodología}

Reconocer la importancia de la educación en el progreso económico nacional es un elemento principal en el propósito de realizar una investigación que pretenda exponer la efectividad de los modelos de desarrollo nacional, en contraste con los resultados educativos en forma de datos duros para su análisis práctico.

Al evaluar los cambios elementales de la política educativa y económica y su posterior impacto en los resultados del sistema educativo nacional, se pueden considerar las conclusiones del trabajo como elemento para la promoción de un cambio que sea más eficaz en los procesos de planificación e implementación de políticas educativas.

La Secretaría de Educación Pública (SEP) en México presenta una serie de estadísticas históricas sobre las principales variables del proceso formativo educativo nacional, tales como la matrícula educativa, las plazas de profesores y el crecimiento de centros educativos a nivel nacional. Dichas estadísticas se extienden a los niveles básico, medio superior y superior. Si bien este último nivel no es obligatorio para la población nacional, se considera elemental para el desarrollo económico, ya que a partir de un mayor índice de profesionales se crea una base sólida de capital humano.

El análisis propuesto en el trabajo evalúa dichas estadísticas. En primer lugar, de forma cuantitativa con el fin de obtener la mayor objetividad sobre el problema destacado, ya que nos referimos a la evolución de la estructura educativa nacional en México. En segundo lugar, de forma cualitativa para contrastar la realidad efectiva del país y corroborar si esto responde o no a las necesidades de la población mexicana, tanto a nivel de promoción social, como en el desarrollo económico y en la integración al aparato productivo nacional.

\section{Crítica a los modelos educativos tradicionales}

La estructura del modelo educativo tradicional ha sido ampliamente criticada a partir de la década de los sesenta, debido a que en esta década se integran una serie de corrientes de pensamiento que dieron paso a dos vertientes. Una de estas aboga por el proceso de desescolarización mediante la clausura de las escuelas como entidades centralistas del conocimiento, sustituyéndolas por la autoeducación en casa o a través de modelos más universalistas. La otra, de corte reformista, la pretende un proceso de integración que no separe los procesos humanistas de las necesidades económicas.

\section{La desescolarización}

El proceso sobre la crítica desescolarizante se presenta en sociedades altamente industrializadas durante la década de los sesenta, momento en que la masividad de la radio y la televisión confluían con los primeros ordenadores con chips integrados. Por tanto, es una crítica con un enfoque altamente tecnológico.

Marshall McLuhan señala la centralización del proceso educativo en el libro. Cualquier otro medio de información es incidental, lo cual contribuye a fomentar, por un lado, la individualidad del estudiante a través del aislamiento (no puede consultar otros medios), y, por otro, la estructura de industrias 
basadas en la apropiación de los derechos para la explotación de esta necesidad.

Sin embargo, el proceso histórico ha demostrado que la enseñanza no siempre estuvo relegada al aprendizaje de los libros. Antes no existían medios para que todos los estudiantes tuvieran un libro, de manera que la observación y el autoaprendizaje formaron parte fundamental del proceso de formación académica. Existía por parte de los individuos un verdadero interés por descubrir la forma en qué se constituía el conocimiento y esto creaba las condiciones para la formación de nuevas ramas pedagógicas (McLuhan).

El proceso histórico en la creación de planes académicos formalizó el estudio y estableció medidas y patrones para el correcto desarrollo de los estudiantes a través del aprendizaje de libros y manuscritos, pero al mismo tiempo ha impedido la apropiación y utilización de las nuevas tecnologías de transmisión de información para el beneficio de la educación (McLuhan).

Los estudiantes veían superados sus conocimientos a través de la información que recibían a diario por todos los medios que los rodeaban, lo cual demostraría como la escuela se rezagaba como centro de difusión del conocimiento si no integraba en sus planes de desarrollo el aprovechamiento de las vías de comunicación masiva y todos los elementos que se desprenden de ellas.

Por otro lado, desde la perspectiva de Iván Illich, el consumismo ha convertido a la escuela en una industria que provee reconocimientos o diplomas como sinónimo de competencia, y cuyo único objetivo es hacer sobrevivir una vasta cadena de burocracia a través de los altos niveles de presupuesto que se le entrega. El aprendizaje es un proceso monopolizado por el profesor, el cual lo abstrae de un contenido previamente estructurado con el fin de discernir la manera correcta de aprender.

Illich señala la escuela como un ente insertado en una sociedad que responde a las necesidades económicas y políticas de un mundo cada vez más globalizado. Las estructuras del conocimiento son una respuesta a las relaciones comerciales. Mientras mayor peso tenga la comunidad en la que se vive, se gozará de mayores prestaciones académicas. Es decir, la importancia productiva de las regiones en un país condiciona las facilidades para que sus habitantes accedan a mayores niveles de educación.

El método institucional tradicional se vuelve un proceso de mantenimiento de la jerarquización social, basado no en las propias capacidades, sino en los medios a los que se puede acceder dependiendo de las condiciones sociales; una cuestión inequitativa y desigual.

El método de enseñanza reprueba todo aprendizaje que no está inscrito en su esfera de enseñanza. De esta forma el autoaprendizaje se declara incapaz de cubrir los estándares de enseñanza y por ello es desplazado: por ser improductivo. La diferenciación es un problema para el sistema académico, ya que la uniformidad del conocimiento es la manera de elaborar planes eficaces para la transmisión (Illich).

Illich tiene varios puntos en común con Everett Reimer, el cual propone la creación de redes de aprendizaje y estructuras de conocimiento socialmente compartidas. Reimer aborda la estructura del aprendizaje como un ente separado de la enseñanza tradicional, pues únicamente la especialización es susceptible de este tipo de formación. El ser humano ha aprendido todas las artes que posee a través de un proceso de aprendizaje continuo en la vida, y esto lo ha dotado de la capacidad para transmitirlo sin la necesidad de un centro físico que integre un proceso para todos los individuos, sin considerar las capacidades individuales o las limitaciones particulares.

Su planteamiento invoca la formación de redes de aprendizaje fundadas en la sociedad convivencial, lo cual limitaría la intervención formativa de estructuras de poder tales como empresas trasnacionales $\mathrm{u}$ organismos internacionales (Reimer).

Estas redes estarían estructuradas en relaciones de iguales: aquellos que ya tienen el saber y los que desean adquirirlo. Debido a que estas relaciones no se basarían en la presentación de diplomas o medios de acreditación, cualquier persona en cualquier época de su vida puede acceder al conocimiento que le haga falta para el desarrollo de su actividad. De igual forma, se prestarían las condiciones adecuadas para que todos aquellos que puedan brindar conocimiento avanzado sean capaces de encontrarse con aquellos que desean adquirirlo.

La principal crítica al sistema educativo tradicional se vuelca sobre el peso que recibe la estimulación económica de las escuelas y no de los estudiantes, por lo que se propone que haya un dispendio benéfico del Estado para los alumnos en función inversamente proporcional a sus posibilidades económicas, de manera que a lo largo de su vida estos puedan ocuparlo para cubrir sus necesidades de formación.

Mediante estos procesos se fomenta el carácter autoorganizativo de la sociedad, se evita delegar 
responsabilidades a grandes órganos burocráticos y se ahorraría una gran cantidad del presupuesto para la inversión en otras figuras más importantes de la esfera educativa, como por ejemplo los estudiantes. Desde esta perspectiva, la institución tradicional le da más importancia a la propia estructura que a los alumnos que acceden a ella.

\section{Paulo Freire: crítica pedagógica}

Paulo Freire menciona que el cambio en el modelo educativo no requiere que se clausuren las instituciones. Señala que el principal protagonista en la transformación de este modelo es el enfoque pedagógico del desarrollo educacional.

En su obra La educación como práctica de la libertad, describe el ejercicio educativo como una manera de transformar el mundo, por lo que es un medio efectivo de emancipar a los pobres de su miseria personal, elevando su calidad de vida y con ello ayudándoles a valorar y validar sus derechos (Freire).

Esta práctica es importante por dos motivos: primero, porque se interesa en cubrir las necesidades de aquellos que ya no son aptos para el mercado productivo por la edad avanzada; y segundo, porque Freire demuestra que a través de la educación para adultos se pueden mejorar las condiciones de vida y la capacidad de obtener un trabajo que les permita subsistir de manera digna.

En el modelo educativo tradicional, Freire señala al educador como una entidad fuera del contexto criticable y en el cual no se le podía considerar un elemento en el campo educacional (Freire). Asimismo, la educación enfrenta la concepción técnica de las necesidades comerciales con la cosmología social de aquellas personas que no se integran como individuos productivos a la sociedad. El efecto es una jerarquización que sitúa a los poseedores de los conocimientos técnicos como superiores y poseedores de la verdad, descalificando a quienes no poseen dichos conocimientos (Freire).

\section{Formación del modelo educativo en México}

El establecimiento del Sistema Educativo Mexicano (SEM), se constituyó de forma gradual y espaciada con base en un proceso ideológico progresista fuertemente orientado hacia la formación de individuos con un carácter más productivo y cívico, ligado a un contexto histórico en el que México transitaba de una serie de conflictos internos hacia la unificación nacional, y de una economía casi inexistente hacia una inserción en la comunidad internacional como nación capaz de brindar elementos humanos productivos (Ornelas).

El SEM se estructuró como un nuevo orden ideológico autoimpuesto. Se vinculó el derecho del saber de la formación educativa hacia una finalidad estrictamente productiva, redefiniendo los objetivos de la educación con base en una valorización meramente económica, pero también de calidad civilizatoria/ colonialista, cuestión planteada por José Vasconcelos.

Al imbuir un espíritu de formación educacional como único, sin reconocer la amplia tradición que pervive en el saber tradicional nacional, el SEM manifestó el rechazo de las diversas cosmovisiones de los naturales locales, debido a que esta no se encontraba en consonancia con el discurso de progreso, producción y cultura enarbolado desde el gobierno nacional posrevolucionario.

Sin embargo, no debe entenderse que la finalidad era la de encumbrar una sociedad dirigida a la creación de estructuras más productivas que sociales, ya que en la labor emprendida por Vasconcelos se concibió la educación como necesaria, de manera que además de formar trabajadores eficaces, se tuviera una enorme visión solidaria y humanista que sirviera en la tarea de facilitar el avance en conjunto del país.

La educación moderna, que abarca la gleba, lo mismo que al privilegiado, ha de dar a cada uno, en su breve paso por las aulas, no sólo cierta especialización técnica que lo habilite para sumarse al ejército irremplazable de los trabajadores, sino también una visión general del mundo invisible, construido por valores que se disfrutan sin sentido de rivalidad o competencia con nuestros semejantes. (Vasconcelos 282)

El SEM, en consecuencia, adicionó a la exigencia de los estudiantes un perfil terminal adecuado para la labor productiva, pero también un perfil y juicio crítico para el enfrentamiento al nuevo proceso y orden social.

El modelo educativo nacional evolucionó en un orden aparentemente enfrentado en sus fines. Ornelas señala como durante la creación de la Secretaría de Educación Pública (SEP) se conjugaron los intereses de dos grandes actores, el político y el educativo, con finalidades ampliamente divergentes. Por un lado, el presidente Calles con la cúpula política nacional, y por otra parte, José Vasconcelos y un grupo 
de intelectuales representantes del interés educativo nacional:

Para ambos grupos, la creación de la Secretaría de Educación Pública significaba un paso trascendente en la historia de México. Para los gobernantes simbolizaba la posibilidad de impulsar un proyecto político de gran alcance en el cual el Estado sería la piedra angular - no sólo de las relaciones políticas sino también de las económicas y las sociales- en la reconstrucción y consolidación de la nación. Para Vasconcelos la creación de la Secretaría de Educación Pública expresaba la eventualidad de erigir la nacionalidad sobre bases culturales que dieran cierta identidad homogénea a los mexicanos. (99-100).

Es que la siguiente persona con mayor influencia e impacto en la SEP, después de Vasconcelos, fue Narciso Bassols, quien fue secretario en 1931 (Ruiz). Bassols estableció como principio rector de la educación nacional un orden socialista con tintes marxistas (Bethell), enfrentado enérgicamente con los principios religiosos todavía presentes en el país, y el cual detentaba una actitud más técnica y menos humanista. Su influencia fue importante y en gran medida se conservó y mantuvo como parte del esquema de formación educativo nacional. Sin embargo, la implicación marxista y el consecuente desarrollo de tendencias divergentes al interés capitalista nacional, derivaron en su remoción como secretario de educación (Camp).

Así, pues, si bien el SEM es un persecutor de un proceso más económico que social, en su propio desarrollo histórico se desenvuelve primero bajo un carácter humanista-nacionalista con Vasconcelos $\mathrm{y}$, después, con Bassols, en un proceso de mejoramiento de las capacidades y aptitudes técnicas de los estudiantes, enfocándose más en la parte práctica que en el aspecto humanista.

Sin embargo, el proceso de mayor relevancia en esta época fue el de incorporar al interés gubernamental la educación rural mediante la creación de escuelas primarias rurales, las cuales surgieron de la propuesta de Vasconcelos (esto con el propósito de aumentar el nivel educativo en estas zonas). Aunado a lo anterior, se crearon las Misiones Culturales con el fin de fortalecer los planes educativos rurales, lo cual se logró al incluir una gran cantidad de profesionistas que pretendían ayudar a los habitantes del lugar a establecer procesos de tecnificación y capacitación en sus labores.
Durante el periodo de mayor influencia de Vasconcelos al frente de la SEP (en 1920), se formalizó la dirección que pretendía seguir la institución educacional. Por lo que desde ese año y hasta 1948, con la intermitente pero importante influencia de Bassols, se crearon en las principales ciudades del país nueve planteles universitarios y la propia Universidad Nacional de México ${ }^{1}$. A estos se les deben sumar cinco escuelas técnicas superiores y al menos treinta escuelas técnicas industriales de nivel medio superior (Rangel). Todas estas escuelas se regían por un programa oficial elaborado desde la SEP que, además de mantener un enfoque humanista, se destacaba por ser laico y gratuito con grado constitucional.

Para apoyar la labor docente, al mismo tiempo que la estudiantil, el gobierno creó comedores, internados, guarderías y becas para el soporte de estudiantes y docentes. En este mismo periodo se formaliza el interés del proceso técnico en las labores profesionales, por lo que se crea el Instituto Politécnico Nacional (IPN) en 1936.

Gran parte de este proceso de expansión y desarrollo de la educación en México es conocido como educación socialista, e inicia en 1934 con la presidencia de Lázaro Cárdenas. Algunos objetivos de esta reforma educativa señalaban que la educación debía ser puerta para integrar las soluciones educativas al problema social de México, convirtiéndola en un actor más activo del proceso nacional (Gilly).

Es así como el nacionalismo permeó todo el proyecto educativo nacional, el cual fue reconocido como un proceso único en el desarrollo de los modelos educativos en Latinoamérica. Sin embargo, no tuvo más relevancia que la de su propia vigencia en el sexenio de Lázaro Cárdenas (oEI).

Gran parte de lo sucedido en materia educativa en México, desde 1940 hasta 1980, puede ser resumido como un proceso que se orientó a cubrir en todo lo posible la educación de la mayor parte de la población en el nivel básico, algo muy significativo para un país en pleno proceso de crecimiento industrial. De forma que los efectos más evidentes fueron el crecimiento notable de la matrícula escolar, así como de la propia cantidad de infraestructura dedicada a la cuestión educativa.

Dicho periodo histórico se desvincula formalmente de lo ocurrido despues de la década de los ochenta, en razón al cambio en el modelo económico

Renombrada como Universidad Nacional Autónoma de México (UNAM) en 1945. 
nacional, lo cual diverge del interés universalista llevado hasta el momento y limita de forma gradual la cantidad y calidad de la educación a la que los mexicanos podrían acceder. Esto se observará y analizará en las siguientes tablas.

Tabla 1. Número de alumnos por nivel educativo (1980-1990)

\begin{tabular}{|c|c|c|c|c|c|c|}
\hline & Preescolar & Primaria & Secundaria & $\begin{array}{c}\text { Media } \\
\text { Superior }\end{array}$ & Superior & Total \\
\hline 1980 & $1,071,619$ & $14,666,257$ & $3,033,856$ & $1,180,135$ & 731,291 & $20,683,158$ \\
\hline 1981 & $1,376,248$ & $14,981,156$ & $3,348,802$ & $1,363,695$ & $7,854,19$ & $21,855,320$ \\
\hline 1982 & $1,690,964$ & $15,222,916$ & $3,583,317$ & $1,535,434$ & 840,368 & $22,872,999$ \\
\hline 1983 & $1,893,650$ & $15,376,153$ & $3,841,673$ & $1,627,518$ & 879,240 & $23,618,234$ \\
\hline 1984 & $2,147,495$ & $15,219,245$ & $3,969,114$ & $1,744,883$ & 939,513 & $24,020,250$ \\
\hline 1985 & $2,381,412$ & $15,124,260$ & $4,179,466$ & $1,897,236$ & 966,384 & $24,548,758$ \\
\hline 1986 & $2,547,358$ & $14,994,642$ & $4,294,596$ & $1,936,077$ & 988,078 & $24,760,751$ \\
\hline 1987 & $2,625,678$ & $14,768,008$ & $4,347,257$ & $2,012,268$ & 989,414 & $24,742,625$ \\
\hline 1988 & $2,668,561$ & $14,656,357$ & $4,355,334$ & $2,070,471$ & $1,033,207$ & $24,783,930$ \\
\hline 1989 & $2,662,588$ & $14,493,763$ & $4,267,156$ & $2,091,920$ & $1,069,565$ & $24,584,992$ \\
\hline 1990 & $2,734,054$ & $1,440,1588$ & $4,190,190$ & $2,100,520$ & $1,078,191$ & $24,504,543$ \\
\hline
\end{tabular}

Fuente: elaboración propia con datos del Sistema Nacional de Información Estadística Educativa, SEN 1893-2012.

En la tabla 1 se observa el desempeño destacable de la educación preescolar, pues su crecimiento casi se triplicó de 1980 a 1990. Dicha característica no la presentó ningún nivel de educación en esta etapa, lo cual responde a los esfuerzos de mejorar la base educativa con el fin de generar un mejor aprovechamiento en los niveles básicos, tema que fue planteado en el Plan Nacional de Educación (PNE) de José López Portillo (1981), quien declaró que esta etapa era prioritaria (Solana).

La tasa anual de crecimiento del número de alumnos para esta década fue de $1,7 \%$, lo que establece un patrón de inefectividad en las políticas educativas y debió de haber marcado un cambio en el paradigma social sobre el modelo educativo tradicional aplicado hasta entonces. Sin embargo, a pesar de esta situación, existió un logro destacable en este periodo, ya que disminuyó el número de personas sin educación en el país, debido principalmente al amplio crecimiento de escuelas durante la década de los cincuenta y sesenta.

En la década de los ochenta, se dio una descentralización de la educación y se realizaron grandes esfuerzos por mantener un nivel más efectivo en el abatimiento del analfabetismo, pero la disminución del presupuesto educativo a raíz de la crisis de la deuda afectó sensiblemente los planes. No solamente perjudicó de forma negativa la matrícula escolar, también afectó al programa de crecimiento estructural educativo, ya que la cantidad de escuelas construidas se detuvo y aún existían más de veinte mil que no habían sido completamente terminadas (Álvarez).

Desde la década de los ochenta, la tendencia educativa en México respondería al paradigma económico neoliberal. Los acuerdos educativos y las sucesivas reformas beneficiarían las relaciones económicas sobre las necesidades sociales, lo que provocó un amplio interés de las corporaciones internacionales por acceder a la mano de obra barata (Banco Mundial).

El cambio del modelo de Industrialización por Sustitución de Importaciones (ISI), al neoliberal, fomentó en el gobierno una necesidad de transitar del interés humanista en la educación al desarrollo técnico (Delors).

Este cambio en la orientación del proceso educativo nacional fue concebido en primera instancia como un método cuyo fin era disminuir la creciente demanda de espacios para los nuevos estudiantes, lo que se hizo a través de exámenes de selección en el ingreso a la educación pública; ${ }^{2}$ después, los resulta-

\footnotetext{
$2 \mathrm{Al}$ respecto, Garduño señala que existe una estandarización obligada en la creación y ejecución de los exámenes, pues en ese proceso, además de racionalizar el acceso a los bienes públicos, también se normaliza y encauza a los estudiantes hacia un fin común de integración social y económica.
} 
dos deficientes de dichos exámenes se vincularon a los propios alumnos y profesores disminuyendo la responsabilidad del Estado en estos problemas (Aguilar; Garduño).

Estos procesos conducen al falso establecimiento de nuevas necesidades educativas, por lo que se propuso la estimulación técnica como medida para superar las dificultades económicas e insertarse correctamente en el mercado laboral, lo que causó detrimento de las carreras de corte humanista, ya que se cree que no son necesarias para las nuevas relaciones comerciales en las que está envuelto el país.

Tabla 2. Número de profesores por nivel educativo (1980-1989)

\begin{tabular}{|c|c|c|c|c|c|c|}
\hline & Total & Preescolar & Primaria & Secundaria & Media Superior & Superior \\
\hline 1980 & 104,144 & 12,941 & 76,024 & 8,873 & 2,908 & 892 \\
\hline 1981 & 112,507 & 17,367 & 76,291 & 11,888 & 3,405 & 938 \\
\hline 1982 & 121,491 & 23,305 & 77,900 & 12,914 & 3,738 & 1,039 \\
\hline 1983 & 128,571 & 28,245 & 78,903 & 13,590 & 3,813 & 1,305 \\
\hline 1984 & 131,317 & 31,022 & 76,183 & 14,789 & 4,952 & 1,540 \\
\hline 1985 & 137,982 & 35,649 & 76,690 & 15,657 & 5,441 & 1,717 \\
\hline 1986 & 147,847 & 40,843 & 80,045 & 16,513 & 5,800 & 1,796 \\
\hline 1987 & 149,267 & 41,438 & 79,677 & 17,640 & 5,586 & 1,930 \\
\hline 1988 & 153,958 & 43,210 & 81,346 & 18,516 & 5,832 & 1,999 \\
\hline 1989 & 154,049 & 43,399 & 80,636 & 18,686 & 6,011 & 2,077 \\
\hline
\end{tabular}

Fuente: elaboración propia con datos del Sistema Nacional de Información Estadística Educativa, sEN 1893-2012.

La justicia social representativa del proceso educativo que se encontraba vigente antes de cambiar al modelo neoliberal se vio desplazada por una tendencia cuantitativa. Se promovió la imagen de como un mayor incremento de egresados capacitados demuestra una mayor calidad en la educación. No obstante, no se ha ahondado en las deficiencias educativas que presentan estos alumnos, así como tampoco en los problemas que tienen para encontrar trabajos de calidad acordes con su nivel de preparación.

Se establecieron mecanismos que fomentaban la selectividad en la educación a través del Centro Nacional de Educación para la Evaluación (CENEVAL), y la Comisión Nacional de Evaluación de la Educación
Superior (CONAEva), lo que confirió a las dos comisiones un carácter de mejoramiento en el proceso de desarrollo de los niveles educativos medio superior y superior (ANUIES).

Este proceso limitó considerablemente la integración de alumnos a la matrícula escolar, disminuyéndola en términos absolutos y promoviendo la idea de incapacidad para acceder a la educación media superior y superior por falta de preparación o capacidad intelectual. Es así que, desde la década de los setenta, los niveles de efectividad en los programas educativos quedan ampliamente limitados (incluso la capacidad para disminuir el analfabetismo disminuyó), tal como se observa en la tabla 3.

Tabla 3. Nivel educativo de la población nacional con más de 15 años (1960-2010)

\begin{tabular}{|c|c|c|c|c|c|c|c|}
\hline & $\begin{array}{l}\text { Sin instruc- } \\
\text { ción }\end{array}$ & $\begin{array}{c}\text { Primaria } \\
\text { incompleta }\end{array}$ & $\begin{array}{l}\text { Primaria } \\
\text { completa }\end{array}$ & $\begin{array}{l}\text { Secundaria } \\
\text { incompleta }\end{array}$ & $\begin{array}{l}\text { Secundaria } \\
\text { completa }\end{array}$ & $\begin{array}{l}\text { Media supe- } \\
\text { rior }\end{array}$ & Superior \\
\hline 1960 & 40,1 & 40,3 & 12 & 2,4 & 2,1 & 2,1 & 1 \\
\hline 1970 & 31,6 & 38,9 & 16,8 & 3,4 & 3 & 3,9 & 2,4 \\
\hline 1980 & 13,7 & 23,2 & 19,7 & 6,3 & 14 & 14,6 & 8,5 \\
\hline 1990 & 10,3 & 18,1 & 19,4 & 5,3 & 19,1 & 16,8 & 11 \\
\hline 2000 & 8,5 & 14,6 & 17,9 & 4,9 & 21,5 & 19,5 & 13,1 \\
\hline 2010 & 6,9 & 11,1 & 18,5 & 4,2 & 23,9 & 20,7 & 14,7 \\
\hline
\end{tabular}

Fuente: elaboración propia con datos del SEN (varios años), INEGI censo de población y vivienda (varios años). 


\section{Educación y pobreza, una relación no antagónica}

Con base en lo anterior, es posible constatar diversas particularidades del universo educativo nacional. Primero, bajo la estructura normativa que rige los principios económicos en los que se fundamenta el país, se transitó hacia un modelo político/económico neoliberal, lo cual dió como resultado una separación y marginalización de los principios humanistas y del incremento en la calidad de la preparación profesional, implantados por Vasconcelos y Bassols.

En segundo lugar, ante el cambio económico e ideológico nacional, el SEM recupera con mayor fuerza uno de sus objetivos principales: servir para cimentar al Estado como elemento rector de las relaciones productivas y económicas del país (Ornelas). Por tanto, a través de la ejecución del proyecto educativo como parte del modelo neoliberal, se establece y regula una conducta afincada en un orden que busca generar mayor eficiencia productiva. No obstante, las continuas crisis económicas, así como los recurrentes problemas en la industria nacional, suelen ser endilgados a la falta de preparación y capacitación de la población.

En tercer lugar, la característica más relevante del proceso educativo nacional desde finales de la década de los setenta hasta la actualidad, es que se ha beneficiado de forma económica y en cantidad de recursos materiales a las escuelas e institutos de niveles educativos medio superior y superior ubicados en zonas urbanas, en contraste con los que se encuentran en áreas rurales o lejanas a las ciudades más importantes del país (Muñoz), a pesar de que estos niveles no son obligatorios. Asimismo, en estos espacios se concentra principalmente la población mexicana con mayor índice de capacidad económica. Sin embargo, esto no genera un mayor aprovechamiento de la población beneficiada, ya que actualmente los logros académicos de estos niveles presentan serias deficiencias en contraste con las de países desarrollados, lo que responde principalmente a la insuficiencia distributiva de los servicios y capacitación que el sistema educativo nacional puede otorgar (Muñoz; Miranda e Islas).

Con base en lo anterior, se puede considerar que la nueva articulación educativa pone énfasis en crear capital humano especializado, dirigiendo el interés hacia la creación de centros especializados para una cantidad y un tipo de población definida con base en sus características económicas y geográficas, lo cual se enfrenta a la postura de Vasconcelos, quien optaba por un mayor nivel de educación.

Es que la nueva dinámica educativa no encara el problema de fondo: el de brindar educación universal y gratuita a toda la población nacional, en parte por dificultades económicas para cubrir la demanda, pero también por el histórico rechazo a las comunidades indígenas.

El SEM abandonó su centralización económica después de la década de los cincuenta. Sin embargo, no ha dejado de centralizar el proceso educativo en el medio urbano, lo que da como resultado una población que en términos prácticos no es mayoritariamente analfabeta, pero que Muñoz reconoce como "analfabetas funcionales", en razón a su incapacidad para acceder a medios que los preparen de cara a las complejas relaciones sociales y productivas actuales.

De acuerdo con Muñoz, se reconoce como gran parte de la deserción estudiantil no solo responde a la dificultad económica para concluir de forma satisfactoria los estudios mínimos, sino también se basa en la falta de recursos que tienen los centros escolares para establecer un mínimo esfuerzo en la capacitación docente de los niveles más bajos de la educación, con la finalidad de que estos puedan motivar a los alumnos a seguir con sus estudios.

Considerando esta situación se puede perfilar lo siguiente:

La educación más deficiente genera mano de obra incapaz de enfrentarse en igualdad de condiciones a cualquier tipo de competencia laboral. Por otra parte, con base en los estándares actuales de trabajo, la educación básica es considerada insuficiente, por lo que existe una absorción limitada de mano de obra en los estratos socioeconómicos más bajos y en el largo plazo la preparación educativa se vuelve algo inútil para su supervivencia.

La educación como medio para evitar la pobreza es un dicho común, se ha repetido incansablemente a través de todos los medios posibles. ${ }^{3}$ No es una relación directa el que a partir de la educación sea posible evitar caer en la pobreza. En México, más de la mitad de la población se encuentra en situación de pobreza, pero menos del $8,5 \%$ de esta es analfabeta, por lo que no resulta sorpresiva la actitud de los jóvenes al

En el Programa Nacional de Educación (DOF) se expone de la siguiente manera: "La prioridad otorgada a la educación básica corresponde a un reclamo permanente de la sociedad: para los mexicanos, una educación pública laica, obligatoria y gratuita constituye el medio por excelencia para el mejoramiento personal, familiar y social." 
descartar la educación como un proceso que les permita salir de esa situación.

La pobreza es un fenómeno social complejo, regularmente definido a partir de una serie de procesos que pretenden "medir" la calidad de vida de las personas mediante encuestas, ${ }^{4}$ índices de nutrición y capacidad para acceder a bienes básicos en relación con el sueldo que perciben. Por otro lado, la educación es fácilmente cuantificable en estadísticas de ingreso y egreso escolar sin considerar sus condiciones sociales. En este sentido, no existe una relación causal entre el nivel de educación con respecto a la miseria en la que se vive. Además de que generalmente es un hecho común el que los lugares geográficos en el país que presentan mayores niveles de pobreza tienen dos características: 1) efectivamente no poseen escuelas de calidad o con niveles superiores a los básicos, y 2) el tipo de empleos existentes no son los adecuados para una persona con preparación.

De esta forma, resulta paralela la existencia de pobreza y deficiencia en la educación, pues en la medida que la segunda no pueda resolver en el corto plazo la situación de miseria en la que se vive, tampoco puede parecer práctico fomentarla. La educación crea capital humano, el cual se encuentra preparado para competir por mejores salarios y generar mayor productividad. Sin embargo, el procedimiento universalista e incluyente impulsado por el gobierno de México no parece considerar la diversidad de realidades sociales y las necesidades en las que confluyen unos y otros. Es que no se conciben planes de estudio diferenciados que consideren las polaridades sociales existentes y fomenten actitudes de permanencia y trabajo en las comunidades.

De acuerdo con Bracho, las personas que viven en situación de pobreza se ven limitadas en sus posibilidades de acceder a la educación, tanto por sus características geográficas como por su posición social. No obstante, aun los que tienen la oportunidad de ingresar a la escuela enfrentan un proceso plagado de problemas; análogo a su propia condición, los pobres son educados pobremente.

Aunado a esto, los sectores con un nivel económico medio también se ven seriamente afectados, pues en la medida en que se configura la especialización, se dificulta el mejoramiento de la calidad de

4 El sitio http://bdsocial.inmujeres.gob.mx/bdsocial/index.php presenta una amplia cantidad de encuestas que evalúan calidad de vida entre otros aspectos (consultado el 10 de mayo del 2015). la enseñanza en niveles básicos, incluso en regiones urbanas con ingresos medios-altos.

Es así que los estudiantes que se encuentran en esta situación, al tratar de ingresar a nivel medio superior educativo, se enfrentan a problemas que desconocen, mientras que a los que egresan también se les dificulta seguir e ingresar al nivel superior educativo. Sin embargo, este filtro no garantiza que al terminar la educación superior se mejore la perspectiva en la calidad de vida, pues como lo menciona Muñoz, este problema da como resultado una espiral inflacionaria de las cualificaciones, lo cual permite a los empleadores disminuir el salario de los más preparados y rechazar a los que se encuentren menos preparados, a pesar de adecuarse a los perfiles laborales.

Silvia Schmelkes elabora una reflexión sobre el proceso y el modelo educativo aplicado actualmente:

Difícilmente los que nos dedicamos a la educación estamos dispuestos a aceptar que la educación es impotente frente a la pobreza; difícilmente accedemos a admitir que la actividad educativa carece de la capacidad de contribuir a la creación de una sociedad más justa, a la formación de individuos autónomos, creativos y participativos y al mejoramiento del nivel de vida de la colectividad social en la que se encuentran insertos los beneficiarios del hecho educativo. (13).

En el modelo educativo tradicional, primero se resuelven las necesidades del aparato productivo, después las necesidades económicas del Estado $y$, finalmente, se intenta resolver el problema social de los individuos que residen en la nación. Es decir, el planteamiento de la educación y su ejecución son el problema de fondo, debido a que se vincula a los individuos con una realidad donde deben coexistir la pobreza y las personas preparadas, pues su trabajo no es disminuir la pobreza sino aumentar el beneficio económico para las empresas y el Estado.

De acuerdo con Bazdresch, el resultado de la aplicación del actual programa educativo en México no considera la disparidad de elementos sociales que coexisten, extendiéndose pero no limitándose a todos los individuos enlistados, lo que tendría como consecuencia problemas de aprovechamiento y alcance en los objetivos de los cursos planteados.

Uno de los resultados de la aplicación del programa educativo mexicano es la deserción y la culminación del ciclo formativo de una manera deficiente, lo que implica que quienes forman a los individuos poseen deficiencias que heredan a los alumnos. La 
calidad académica es también un factor que precisa atención, ya que en algunos casos a causa del bajo monto en los salarios, los profesores tampoco podrán acceder a cursos que los actualicen y preparen de mejor manera.

En suma, la pobreza responde a cuestiones prácticas que requieren respuestas de corto plazo, en tanto que la educación forma a personas con objetivos a largo plazo y encauzadas a insertarse en el mercado de trabajo, con miras a resolver problemas no relacionados directamente con la pobreza.

\section{El proceso de desescolarización en México: ¿una opción viable?}

En el escenario que se ha planteado hasta ahora, es importante considerar la desavenencia del modelo educativo aplicado en relación con los resultados obtenidos, por lo cual se debe recuperar la crítica al modelo educativo tradicional. De acuerdo con Esterle, la desescolarización es considerada como una expresión del fracaso escolar, de falta de capacidad del Estado para afrontar el problema y un proceso indebido de planeación de las necesidades sociales presentes y futuras.

Por lo tanto, el modelo de desescolarización plantea un escenario en el cual la institución pública fracasa en su objetivo, de manera que irremediablemente atrae los primeros objetivos de la SEP a nivel nacional sobre una alfabetización en todo el país $y$, sin embargo, a casi 100 años de que se haya proyectado, aún sigue sin poder lograrse. Por esto es necesario preguntarse si el sistema educativo tradicional mexicano sigue siendo vigente ante los resultados expuestos y, sobre todo, al considerar la posibilidad de una educación alternativa basada en la teoría de la desescolarización.

El movimiento de desescolarización surge como parte de un proceso histórico, en el cual confluye el interés por el avance tecnológico con una profunda crisis económica en Estados Unidos ${ }^{5}$. En este escenario se difundió la idea según la cual el proceso de escolarización tenía un importante desfase frente al desarrollo técnico, de modo que se tornaba obsoleta en términos prácticos. Los planes de estudio, así como

Dicha crisis se genera por los conflictos internacionales en Medio Oriente que encarecen el precio del petróleo, así como por una pérdida de competitividad ante Europa y Asia. Momento que es aprovechado para realizar una crítica a la política de beneficio público de Estados Unidos. las reformas más importantes, tardan mucho tiempo en aplicarse al programa vigente y dan como resultado un conocimiento desfasado del interés social. Por otra parte, el proceso para formar nuevas generaciones es muy lento (casi veinte años hasta cubrir la educación superior), y no se puede garantizar que las generaciones educadas tendrán éxito en el desarrollo social. Esto representaba una fuga de capital que bien podría ocuparse para otro tipo de formación educativa elegida a su conveniencia.

Como ya se planteó en el segundo apartado, uno de los principales intereses al crear la SEP fue el de inculcar a la población mexicana la noción de un Estado mexicano como rector de las relaciones públicas, sociales y económicas. Esto se podría lograr al emprender una formación educativa de largo plazo, regida y mantenida por un organismo central que se estructuró con base en los intereses políticos y económicos del propio Estado. Lo anterior tuvo un aparente beneficio durante las primeras etapas de la SEP, pero debido a la propia necesidad de ejercer desde el interés y conveniencia del Estado se vio entorpecida su labor, la cual debería tener como principal objetivo educar a la ciudadanía. Asimismo, desde la década de los ochenta, dicha secretaría ha creado barreras económicas y sociales a través de exámenes y pruebas de ingreso que no contemplan la realidad social y económica del país.

De esta forma, el sistema educativo nacional ingresó a un proceso reformista, pero no en el sentido del movimiento desescolarizante, sino de forma contraria al interés social, ya que se limitó la capacidad del Estado para brindar educación a toda la población. También, se restringió la capacidad de los ciudadanos para acceder a otras opciones que no fueran las instituciones privadas. Las críticas de Illich y Reimer giran en torno a suprimir la coacción del Estado sobre las posibilidades educativas a las que podrían acceder los alumnos, refrendando en este sentido la capacidad nata de los seres humanos de aprender en otros espacios que no solo sean los proporcionados por el Estado.

México ha transitado hacia un proceso en el cual se legitima el rechazo educativo y la única opción viable - para la población más pobre, es incorporarse al mercado laboral con poca o nula preparación, pues no se cuenta con las capacidades económicas adecuadas para acceder a otro tipo de formación y tampoco se proporciona una alternativa válida ante esta situación. 
Illich propone privar de apoyo económico a la escuela, ya que su carga económica en el largo plazo es muy alta, sobre todo al considerar que gran parte de lo gastado se dirige hacia costos administrativos y burocráticos. México destina más del 5\% del PIB al proceso educativo nacional. Sin embargo, este proceso no se ve retribuido en la medida que la escuela pueda vencer a la pobreza constante que existe en el país. Como alternativa a este enorme dispendio económico por parte del Estado en una actividad que no brinda resultados efectivos contra la pobreza y otros malestares sociales, Ilich proponía una renta educativa para cada familia con hijos, la cual pudieran usar tanto en educación privada como en educación en casa. Sin embargo, con base en las cifras de la Cepal', el gasto público social por habitante en cuestión educativa en el país fue de 300 dólares en el 2012, por lo que es difícil pensar que se pueda brindar una educación de mejor calidad que la que ofrecen los centros educativos actuales. Por otra parte, la población que se encuentra en la peor situación económica está laborando en trabajos que les exigen horarios sobresaturados, lo que impediría una formación educativa adecuada desde casa.

La propuesta de Ilich puede ser viable siempre y cuando el Estado proporcione a la población una cantidad de dinero razonable para este gasto. Además, los beneficiados deben tener tiempo suficiente. Estas son cuestiones que por el momento no se visualizan en el actual escenario económico y político del país. Por otro lado, debido a que no existe una ley actualmente que acredite la enseñanza desde casa, es complejo que las universidades y las empresas acepten a quienes hayan sido educados de esta forma.

Con lo anterior no se pretende decir que la educación en México se deba mantener como se encuentra actualmente. Es que si bien no existen marcos normativos para la educación en casa, esta es una iniciativa que tiene una notable cantidad de apoyo ${ }^{7}$ y puede acceder a un proceso de revalidación, a partir del cual se permita a las personas obtener el grado de enseñanza básica y media sin tener que asistir a una institución púbica.-

Se puede mencionar que estas cuestiones tienen relevancia porque la educación en México ha

\footnotetext{
6 Datos disponibles en CEPALSTAT: http://estadisticas.cepal org/cepalstat/WEB_CEPALSTAT/estadisticasIndicadores.asp?idioma $=$ e (Consultado el 23 de julio de2014).

No existen estudios sobre el tema, pero hay una gran cantidad de resultados en Internet sobre asociaciones civiles que se dedican al desarrollo y capacitación para la educación en casa.
}

demostrado ser ineficaz para cubrir los niveles básicos de educación. Además, cuando se logra que los estudiantes cubran dichos niveles educativos, suelen presentar una carencia de capacidades en comparación con otros países. Lo anterior lleva a suponer que el problema radica en que no existe un adecuado encausamiento de recursos económicos dedicados a este rubro y a la ausencia de capacitación educativa constante para los docentes. La escuela como institución pública en México sigue siendo importante, y su existencia no debería ser excluyente de otras opciones como la educación en casa. Es necesaria una reforma educativa que consista en generar mejores condiciones y más opciones, tanto para los estudiantes, como para los profesores.

\section{Conclusiones}

La educación libre, gratuita y universal en México comienza hasta el segundo cuarto del siglo $\mathrm{xx}$, por lo que su desarrollo ha tenido una gran cantidad de contrastes que no pueden ser analizados de forma separada.

El proceso educativo nace del verdadero interés por crear una base de superación para todos, lo que además impulsa la capacidad de desarrollo y solidaridad. Sin embargo, en el caso de nuestro país, dicho proceso responde análogamente a las necesidades de la base industrial, por lo que, si bien las ideas e intenciones originales son humanistas, su ejecución es de interés más industrial y elitista, ya que favorece la formación de unos cuantos en razón a la inviabilidad económica que supone beneficiar al total de la población que demanda educación.

A través de la historia de la educación en México, se observa como a pesar del esfuerzo que se ha hecho por aumentar la capacidad estructural y docente, ha sido inviable en el largo plazo, ya que la mayor parte de los estudiantes que renuncian a la escuela lo hacen, entre otras razones, a causa de los pocos recursos económicos con los que cuentan para asistir a la escuela, o bien por la limitada cantidad de materiales que existen en ella para atender las necesidades básicas de educación de una gran cantidad de alumnos. Por otro lado, las instituciones sufren deterioro, tanto por la sobreutilización, como por la falta de fondos para cubrirlos.

Asimismo, se puede observar un modelo educativo incapaz de responder a la demanda, desfasado en el tiempo y nulo en utilidad ante los nuevos paradigmas sociales. Es necesario hacer un análisis de fondo 
sobre los objetivos que se pretenden cubrir y cumplir, pues únicamente crecer en cobertura en algunos niveles tampoco ha servido para disminuir la pobreza y marginación en México.

A través de los resultados mostrados se puede abordar una perspectiva que pone en duda la actual relevancia del SEM, no tanto por la parcialidad de su ejecución, sino por la incapacidad que tiene para adaptarse al cambio en el proceso educativo actual, a las exigencias de los nuevos empleos y por ser incapaz de conjugar una relación entre capacidad productiva y desarrollo humano.

Por otro lado, no debe rechazarse la perspectiva de una educación que se ha brindado con cierta efectividad durante varias décadas en México. Sin embargo, se puede establecer que el resultado actual de la educación en el país es un proceso de burocratización, en el cual se limita la calidad de la enseñanza, desalienta la formación y actualización de los profesores en los niveles más bajos, así como margina a los sectores más desprotegidos y menos desarrollados de la nación.

En este sentido, valdría la pena recuperar los planteamientos de McLuhan y los de Freire, ya que es complicado suponer que se puede estructurar desde cero un nuevo planteamiento educativo nacional. Sin embargo, es importante reconocer los resultados actuales y señalar que debe existir un cambio en la centralización urbanizada y económica de la educación, y aceptar que existe un universo social mucho mayor, el cual es necesario para el desarrollo del país.

Por otro lado, los grandes y positivos logros de Freire en su país (Brasil) han demostrado que es posible realizar un cambio en materia educativa sustentado en el propio sistema educativo, sin que tenga que ser este rechazado desde la base.

Finalmente, en la medida que los objetivos educativos respondan a las necesidades sociales inmediatas y logren fomentar un nivel de superación observable en los niveles de pobreza, se podrá considerar vinculante la relación entre la educación y la sociedad.

\section{Referencias}

Aguilar, Luis. La hechura de las políticas. México: Miguel Ángel Porrúa, 2007. Impreso.

Álvarez Mendiola, Germán. Sistema educativo nacional de México. México: Secretaría de Educación Pública y Organización de Estados Iberoamericanos, 1994. Impreso.
ANUIES. La educación superior en el siglo XXI, líneas estratégicas de desarrollo: una propuesta de la ANUIES. México: UNAM, 2000. Impreso.

Banco Mundial. Constructing Knowledge Societies: new challenges for tertiary education. Nueva York: Banco Mundial, 2002. Impreso.

Bethell, Leslie. The Cambridge History of Latin America. Cambridge, Inglaterra: Cambridge University, 1995. Impreso.

Bazdresch, Miguel. "La intervención de la práctica educativa”. La significación de la práctica educativa. Ed. Ruth Perales Ponce. México: Paidós, 2006. 55-69. Impreso.

Camp, Roderic Ai. Mexican Political Biographies, 19351993. Austin, Texas: University of Texas Press, 1995. Impreso.

Delors, Jacques. La educación encierra un tesoro. México: Uniesco, 1996. Impreso.

De Zumbiría Samper, Julián. Los modelos pedagógicos: hacia una pedagogía dialogante. Colombia: Editorial Magisterio, 2006. Impreso.

Diario Oficial de la Federación (DOF). Programa de Desarrollo Educativo 1995-2000. México: Secretaría de Gobernación, 1996. Impreso.

Freire. Educación como práctica de la libertad. España: Siglo xxI Editores, 2009. Impreso.

----, Paulo. Pedagogía del oprimido. México: Siglo xxi Editores, 2005. Impreso.

Garduño, Felipe. "Los exámenes de admisión y la selección a la universidad pública, ¿razón pedagógica o racionalidad técnica?". Revista del Centro de Investigación 4.14 (2000): 11-18. Impreso.

Gilly, Adolfo. El cardenismo, una utopía mexicana. México: Ediciones Era, 2001. Impreso.

Illich, Iván. La sociedad desescolarizada. España: Joaquín Moritz, 1985. Impreso.

Luengo Navas et al. "Las reformas educativas basadas en el enfoque por competencias: una visión comparada”. Profesorado 12.3 (2008): 1-10. Impreso.

McLuhan, Marshall. "El aula sin muros”. El aula sin muros: investigaciones sobre técnicas de comunicación. Barcelona: Laia, 1974:155-156. Impreso.

Miranda López, Francisco y Juana María Islas Dossettí. "Educación media superior en México. Hacia una política de reescolarización”. Revista Iberoamericana de Educación 64 (2014): 1-7. Impreso.

Muñoz. "Construcción del Conocimiento sobre la Etiología del Rezago Educativo y sus Implicaciones para la 
Orientación de las Políticas Públicas: la Experiencia de México". REICE Monográfico: Abandono y Deserción en la Educación Iberoamericana 7.4 (2009): 10-27. Impreso.

---- Izquierdo, Carlos. "El papel de la educación en el desarrollo económico y social; una perspectiva". Revista de la Educación Superior 37 (1981). Impreso.

Organización de Estados Iberoamericanos. Sistema Educativo Nacional de México: 1994. México: Secretaría de Educación Pública, 1994. Impreso.

Organización para la Cooperación y el Desarrollo Económico (OCDE). Knowledge Management in the Learning Society. Francia: Publicaciones OCDE, 2000. Impreso.

Ornelas, Carlos. "Las bases del federalismo y la descentralización en educación". Revista de Investigación Educativa 5.1 (2003): 118-134. Impreso.

----. El Sistema educativo mexicano: la transición de fin de siglo. México: Centro de Investigación y Docencia Económicas, 1995. Impreso.

Rangel Guerra, Alfonso. La educación superior en México. México: El Colegio de México, 1983. Impreso.
Ratinoff, Luis. "La crisis de la educación: el papel de las retóricas y el papel de las reformas". Revista Latinoamericana de Estudios Educativos xxIv.4 (1994): 15-96. Impreso.

Reimer, Everett. La escuela ha muerto: alternativas en materia de educación. Argentina: Labor, 1981. Impreso.

Ruiz, Ramón Eduardo. Triumphs and Tragedy: A History of the Mexican People. NY: W. W. Norton \& Company. 1992. Impreso

SEN. Sistema Nacional de Información Estadística educativa 1893-2012. México, 2012. Impreso.

Schmelkes, Silvia. Educación y pobreza. México: Unicef y Colegio Mexiquense, 1995. Impreso.

Solana, Fernando. ¿Qué significa calidad en la educación? México: Ed. Grupo Noriega, 2002. Impreso.

Vasconcelos, José, Antología de textos sobre educación, MéXico: SEP, 1981. Impreso.

\section{Fuentes de datos estadísticos}

CEPALSTAT. http://estadisticas.cepal.org/cepalstat/WEB_ CEPALSTAT/estadisticasIndicadores.asp?idioma $=\mathrm{e}$ 